\title{
Neutron Structure - New Results with CLAS at Jefferson Lab
}

\author{
Sebastian E. Kuhn \\ Old Dominion University, Norfolk, VA 23529
}

\begin{abstract}
New measurements using the $6 \mathrm{GeV}$ continuous electron beam and the CEBAF Large Acceptance Spectrometer (CLAS) at Jefferson Lab have collected information on the form factors and the unpolarized structure functions of the neutron, with minimal uncertainty from nuclear binding effects. One experiment has also tried to measure these binding effects more directly, using the method of "spectator tagging". These experiments are forerunners for an extensive program with the energy-upgraded $12 \mathrm{GeV}$ accelerator at Jefferson Lab.
\end{abstract}

Keywords: Neutron Structure, Neutron Form Factors, Novel Experimental Techniques

PACS: $14.20 . \mathrm{Dh}, 13.40 . \mathrm{f}, 13.60 . \mathrm{Hb}$

\section{INTRODUCTION}

A truly comprehensive amount of data has been collected on the structure of the proton - its electromagnetic form factors and its structure functions in the deep inelastic and resonance region. Much less is known about the neutron, since free neutron targets of sufficient density are not available and information gathered from nuclear target data requires model-dependent (and often uncertain) corrections for nuclear binding. However, neutron structure data are just as important as those on the proton because only the combination of both lets us extract unambiguously the contribution from both up and down quarks to the size, shape and excitations of the nucleon, and to the momentum fraction carried by its quarks. At Jefferson Lab, several experiments have recently employed new techniques to overcome this impasse and to access structure information for the (nearly) free neutron, as well as possible structure modifications when a neutron is close to another nucleon. Some of these experiments and their results are briefly portrayed in the following.

\section{THE MAGNETIC FORM FACTOR $G_{M N}$ OF THE NEUTRON}

In a recent experiment ("E5"), the magnetic form factor of the neutron was determined by measuring the ratio of the quasi-elastic electron cross section on the neutron to that on the proton inside deuterium. This method leads to a cancellation of systematic errors from acceptance, luminosity and nuclear modifications of nucleon form factors. By detecting the struck nucleon in both cases, the kinematics of the reaction could be restricted to low recoil momenta for the other ("spectator") nucleon, which reduces model uncertainties. The neutron detection efficiency of the electromagnetic calorimeter and ToF scintillator counters was measured simultaneously by tagging neutrons in the

\author{
CP870, Intersections of Particle and Nuclear Physics: $9^{\text {th }}$ Conference, \\ edited by T. M. Liss \\ (c) 2006 American Institute of Physics 978-0-7354-0368-0/06/\$23.00
}




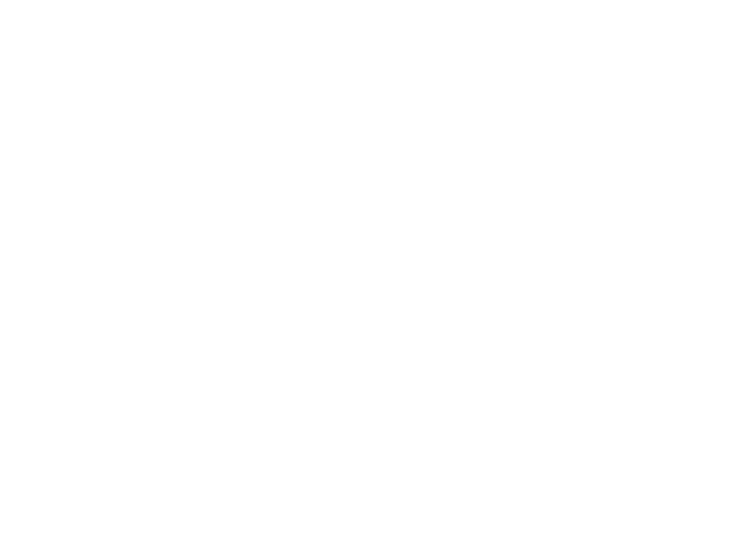

FIGURE 1. Results on the neutron form factor $G_{m}^{n}$, normalized to the dipole form factor $G_{D}$, from the experiment with CLAS.

reaction $p\left(e, e^{\prime} \pi^{+}\right) n$ using a second target filled with hydrogen. Using the very well measured form factors of the proton (and correcting for the very small effect of the electric neutron form factor $G_{E n}$ ), the magnetic form factor of the neutron $G_{M n}$ could be extracted over a wide range in momentum transfer, $1 \leq Q^{2} \leq 4.5 \mathrm{GeV}^{2}$.

Figure 1 shows our results [1], normalized to the usual dipole parameterization of the nucleon form factors. While the data show little deviation from the smooth dipole shape (except for possibly a drop-off at the highest $Q^{2}$ ), their density and precision is far better than for all previous data (some of which are shown in the plot).

\section{NEUTRON STRUCTURE FUNCTIONS - THE SPECTATOR MECHANISM}

Binding corrections for structure functions measured on the neutron in deuterium tend to be larger if in the initial state the two nucleons were close together or, equivalently, had large internal momenta. Therefore, a promising way to either suppress these corrections (for extraction of free neutron structure functions) or to study them in more detail is to require simultaneous detection of a spectator proton whose momentum carries information from the initial state. By selecting protons moving backwards relative to the momentum transfer $\vec{q}$, one can ensure that the reaction took place on the neutron and that the proton was a more-or-less undisturbed spectator in this reaction. One can also use the measured proton momentum $p_{s}$ to infer the initial momentum of the neutron before it was struck, and therefore correct all kinematic variables like $x$ and the final state invariant mass, $W$, for this initial motion. Restricting $p_{s}$ to less than about $0.1 \mathrm{GeV} / \mathrm{c}$ selects the part of the deuteron wave function with large nucleon-nucleon separation and minimal binding effects. Vice versa, if $p_{s}$ is larger than $0.3 \mathrm{GeV} / \mathrm{c}$, the struck neutron is rather far off its mass shell and one is sensitive to short internucleon separations. More details on the theoretical background can be found in Ref. [2]. 


\section{The "Deeps" Experiment}

The "Deeps" experiment used the large acceptance of CLAS and a liquid deuteron target to study inclusive scattering off the neutron with simultaneous detection of a rather fast backward-going proton (above $0.3 \mathrm{GeV} / \mathrm{c}$ ). The goal of the experiment was to study the reaction mechanism (in particular the spectator approximation) and to look for a dependence of the extracted "off-shell" neutron structure function on the spectator momentum.

We found [3] that the momentum distribution for protons moving backward relative to $\vec{q}$ could be well described by the intrinsic momentum distribution of the two nucleons in deuterium (left panel had much larger momer of Fig. 2), consistent wil debris) and the "spectator to increase with $W$. Our shell" neutron structure f
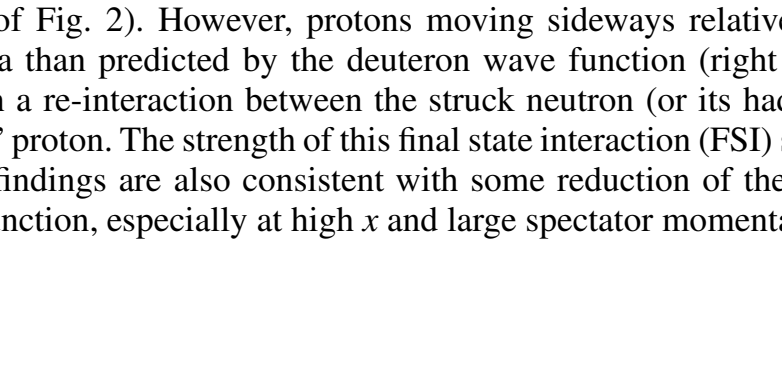

o $\vec{q}$

anel

snic

ams

off$s_{s}$.
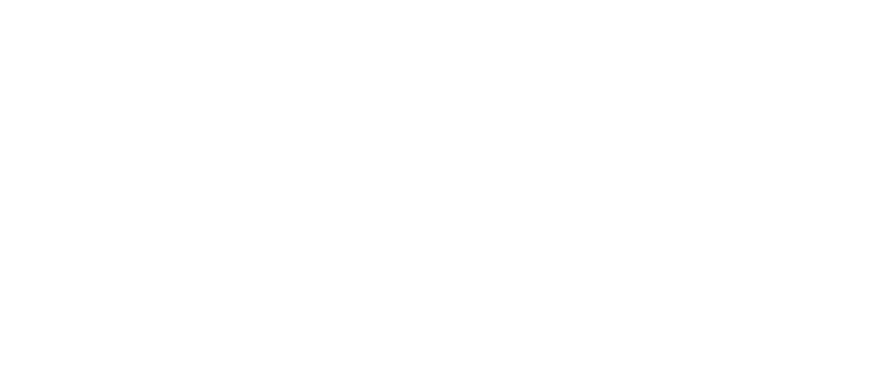

FIGURE 2. Momentum distribution of the observed "spectator" proton in the reaction $d\left(e, e^{\prime} p_{s}\right) X$ for backward angles $\left(\theta_{p q}>108^{\circ}\right.$, left $)$ and for rescattering kinematics $\left(72^{\circ} \leq \theta_{p q} \leq 108^{\circ}\right.$, right $)$. The top panels are for quasi-elastic scattering $d\left(e, e^{\prime} p_{s}\right) n$, the middle panels for an invariant mass $W$ of the unobserved final state $X$ in the resonance region, and the bottom panels for $W>2 \mathrm{GeV}$. The solid lines are from simple PWIA calculations in the spectator picture. 


\section{The "BoNuS" Experiment}

Tagging spectator protons at large backward angles and at low momenta allows us to measure directly the structure function of the "nearly free" neutron, with minimal binding and FSI corrections. For this purpose, the "BoNuS" collaboration built a novel proton recoil detector with a threshold for spectator protons down to $0.07 \mathrm{GeV} / \mathrm{c}$. The detector is immersed in a solenoidal magnetic field and employs a annular drift region (filled with a Helium/DME gas mixture) of $3 \mathrm{~cm}$ depth. The whole proton track in this region is reconstructed in a "TPC-like" fashion, using a 2-dimensional readout of position and drift time on the mantle of the cylinder. Secondary electron amplification is accomplished with 3 cylindrical layers of Gaseous Electron Multipliers (GEMs). The expected results from the run, which concluded last year, are shown in Fig. 3.

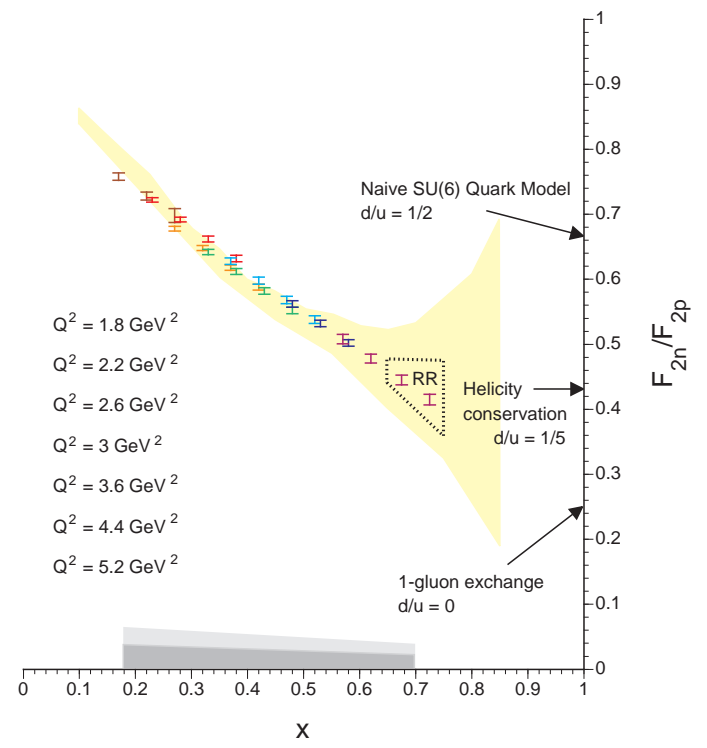

FIGURE 3. Expected uncertainties for the ratio of DIS neutron to proton structure functions, $F_{2 n} / F_{2 p}$, from the "BoNuS" experiment. High precision data were also collected in the resonance region.

\section{ACKNOWLEDGMENTS}

I would like to thank the CLAS collaboration and the technical staff at Jefferson Lab. This research has been supported by the DOE under contract DE-FG02-96ER40960.

\section{REFERENCES}

1. W. K. Brooks, and J. D. Lachniet, Nucl. Phys., A755, 261-264 (2005), nucl-ex/ 0504028.

2. M. M. Sargsian, et al., J. Phys., G29, R1 (2003), nucl-th/0210025.

3. A. V. Klimenko, et al., Phys. Rev., C73, 035212 (2006), nucl-ex/ 0510032. 